\title{
The Type-1 OWA Operator and the Centroid of Type-2 Fuzzy Sets
}

\author{
Francisco Chiclana ${ }^{1}$ Shang-Ming Zhou ${ }^{2}$ \\ ${ }^{1}$ Centre for Computational Intelligence, De Montfort University, Leicester, LE1 9BH, UK. \\ ${ }^{2}$ Health Information Research Unit, School of Medicine, Swansea University, SA2 8PP, UK.
}

\begin{abstract}
This paper aims to establish the relationship between two apparent disparate problems: (i) the aggregation of uncertain information modelled by type-1 fuzzy sets via OWA mechanism, and (ii) the computation of the centroid of type-2 fuzzy sets. In order to cut down the computational complexity of the direct approach to performing type-1 OWA operation, the $\alpha$-level approach to type-1 OWA operators was developed. This new approach is based on the decomposition of a type-1 OWA operator via its $\alpha$-levels and the corresponding Representation Theorem of type-1 OWA operators. A close inspection of the mathematical representation of the centroid of type-2 fuzzy sets reveals that this is a special case of type-1 OWA operator. This relationship will allow for the computation of the centroid of a general type-2 fuzzy sets to be carried out via the application of the representation theorem to its equivalent type-1 OWA representation.
\end{abstract}

Keywords: Type-1 fuzzy sets, type-2 fuzzy sets, type-1 OWA operators, $\alpha$-level, centroid, representation theorem.

\section{Introduction}

The aggregation of information via OWA operators [1] and the defuzzification of a fuzzy set [2] have up to now being treated as different and unconnected problems in Fuzzy Set Theory research. However, a close inspection of their mathematical representation suggests that the second one could be seen as a special case of OWA operator.

Although the defuzzification a type-1 fuzzy sets does not present any challenge from a mathematical point of view, the same cannot be said in the case of type-2 fuzzy sets. For type-2 fuzzy sets, the defuzzification stage consists of two parts [3]: (i) type-reduction - procedure by which a type-2 fuzzy set is converted to a type-1 fuzzy set, known as the type-reduced set (TRS); and (ii) defuzzification proper - the TRS is defuzzified to give a crisp number, the centroid of the type-2 fuzzy set. Obviously, the computation of the TRS is a challenging step in the design of type-2 fuzzy systems. For generalised type-2 fuzzy sets the defuzzification process is complex and its direct implementation is slow and inefficient. This has inevitably hindered the development of type-2 fuzzy inferencing systems for real applications. The present situation will not be expected to change, unless an efficient and fast method to deffuzzify general type-2 fuzzy sets is developed. Indeed, most researchers concentrate exclusively on the development of theoretical results and practical applications for interval type-2 fuzzy sets [4-11].

The above described scenario for the centroid of a general type-2 fuzzy set is remarkably similar to the recent development of the Type-1 OWA operator [12]. Type-1 OWA operators provide us with a new technique for directly aggregating uncertain information modelled by type- 1 fuzzy sets via OWA mechanism in soft decision making and data mining. It is known that aggregation is a necessary step in either multi-expert decision making or multi-criteria decision making [13-15]. Type-1 OWA operators can be used to aggregate expert knowledge expressed by type- 1 fuzzy sets in decision making, and they also have the potential of merging fuzzy sets in fuzzy modelling to improve model interpretability and transparency [16-18]. However, the direct approach to performing type-1 OWA operation involves high computational load, which inevitably curtailed further applications of type-1 OWA operator to real world decision making. To overcome this issue, a new approach to type-1 OWA operations has been developed based on the $\alpha$-levels of fuzzy sets [19]. This result is based on the so-called Representation Theorem of type-1 OWA operators [19], which provides the decomposition of a type-1 OWA operator into a series of its $\alpha$-level type-1 OWA operators. It provides an effective tool for performing type-1 OWA operations. Indeed, the complexity of this $\alpha$-level approach is of linear order, so it can be used in real time soft decision making, database integration and information fusion that involve aggregation of uncertain information.

Mathematically, the centroid of a type- 1 fuzzy set can be seen as the output of an OWA operator applied to a set of crisp values. This means that in practice, the computation process of the centroid of a type-2 fuzzy sets could be carried out by applying its equivalent OWA computation process. The centroid of a type-2 fuzzy set and the type-1 OWA operator were both developed via the application of Zadeh's Extension Principle [20]. We hypothesise that a result connecting the mathematical representation of the centroid of a type-2 fuzzy set and 
the representation of a type-1 OWA operator can be proved. Indeed, we will show that the centroid of a type-2 fuzzy set, as defined in [3], is equivalent to that of a type-1 OWA operator, as defined in [12]. We extend this equivalent mathematical representation to the centroid of interval type- 2 fuzzy sets and the $\alpha$-level type-1 OWA operators.

The main contribution of our research is that the fast and efficient method for computing type-1 OWA operations, the $\alpha$-level Approach, developed in [19] can be applied to compute the centroid of a general type-2 fuzzy set. This would lead to the computation of the TRS of a general type- 2 fuzzy sets via the Type-1 OWA Representation Theorem. As a result, a fast and efficient method of computing the centroid of general type- 2 fuzzy sets can be realised.

\section{Type-1 and Type-2 Fuzzy Sets}

Type-1 Fuzzy Set. Let $X$ be a universe of discourse. A fuzzy set $A$ on $X$ is characterised by a membership function $\mu_{A}: X \rightarrow[0,1]$. A fuzzy set $A$ on $X$ can be expressed as follows:

$$
A=\left\{\left(x, \mu_{A}(x)\right) \mid \mu_{A}(x) \in[0,1] \forall x \in X\right\} .
$$

An alternative mathematical representation of fuzzy set $A$ with continuous universe of discourse is

$$
A=\int_{x \in X} \mu_{A}(x) / x
$$

When the universe of discourse is discrete the fuzzy set $A$ is represented as

$$
A=\sum_{x \in X} \mu_{A}(x) / x
$$

Note that the membership grades of $A$ are crisp numbers. This type of fuzzy set is also referred to as a type- 1 fuzzy set. In the following we will use the notation $U=[0,1]$.

The Representation Theorem of (type-1) fuzzy sets provides an alternative and convenient way to define type-1 fuzzy sets via their corresponding family of crisp $\alpha$-level sets. The $\alpha$-level set of a type- 1 fuzzy set $A$ is defined as

$$
A_{\alpha}=\left\{x \in X \mid \mu_{A}(x) \geq \alpha\right\}
$$

The set of crisp sets $\left\{A_{\alpha} \mid 0<\alpha \leq 1\right\}$ is said to be a representation of the type- 1 fuzzy set $A$. Indeed, the type- 1 fuzzy set $A$ can be represented as

$$
A=\cup_{0<\alpha \leq 1} \alpha A_{\alpha}
$$

with membership function

$$
\mu_{A}(x)=\underset{\alpha: x \in A_{\alpha}}{\vee} \alpha
$$

This is the the so-called 'horizontal' representation of a type-1 fuzzy set.
Type-2 Fuzzy Set. A type-2 fuzzy set $\tilde{A}$ on $X$ is a fuzzy set whose membership grades are themselves fuzzy. This implies that $\mu_{\tilde{A}}(x)$ is a (type-1) fuzzy set on $U$ for all $x$, i.e.

$$
\tilde{A}=\left\{\left(x, \mu_{\tilde{A}}(x)\right) \mid \mu_{\tilde{A}}(x) \in \tilde{P}(U) \forall x \in X\right\} .
$$

where $\tilde{P}(U)$ is the set of fuzzy sets on $U$.

This implies that for all $x \in X$ there exists a subset of $U, J_{x}$, such that $\mu_{\tilde{A}}(x): J_{x} \rightarrow U$. Applying (1), we have:

$$
\begin{array}{r}
\mu_{A}(x)=\left\{\left(u, \mu_{\tilde{A}}(x)(u)\right) \mid \mu_{\tilde{A}}(x)(u) \in U\right. \\
\left.\forall u \in J_{x} \subseteq U\right\} .
\end{array}
$$

$J_{x}$ is called the primary membership of $x$ while $\mu_{\tilde{A}}(x)$ is called the secondary membership of $x$.

Putting (7) and (8) together we have

$$
\begin{gathered}
\tilde{A}=\left\{\left(x,\left(u, \mu_{\tilde{A}}(x)(u)\right)\right) \mid \mu_{\tilde{A}}(x)(u) \in U \forall x \in X\right. \\
\left.\wedge \forall u \in J_{x} \subseteq U\right\} .
\end{gathered}
$$

Interval Type-2 Fuzzy Set. An interval type-2 fuzzy set is a type-2 fuzzy set with constant secondary membership function 1, i.e. $\mu_{\tilde{A}}(x)(u)=$ $1, \forall u \in J_{x}$.

\section{Type-1 OWA Operators}

In 1988, Yager introduced an aggregation technique based on the ordered weighted averaging (OWA) scheme [1].

Definition 1 An OWA operator of dimension $n$ is a mapping $\phi: \mathbb{R}^{n} \rightarrow \mathbb{R}$, which has an associated set of weights $\left\{w_{i}\right\}_{i=1}^{n}$ to it, so that $w_{i} \in[0,1]$ and $\sum_{i=1}^{n} w_{i}=1$

$$
\phi(a)=\phi\left(a_{1}, \cdots, a_{n}\right)=\sum_{i=1}^{n} w_{i} a_{\sigma(i)}
$$

where $\sigma:\{1, \cdots, n\} \rightarrow\{1, \cdots, n\}$ is a permutation function such that $a_{\sigma(i)}$ is the $i$-th highest value in the set $\left\{a_{1}, \cdots, a_{n}\right\}$, i.e. $a_{\sigma(i)} \geq a_{\sigma(i+1)} \forall i$.

Generally speaking, the OWA operator based aggregation process consists of three steps: (i) the first step is the re-ordering the input arguments in increasing order. In this way, a particular element to aggregate is not associated with a particular weight, but rather a weight is associated with a particular ordered position of an aggregated object; (ii) the second step is to determine the weights for the operator in a proper way; (iii) finally, the OWA weights are used to aggregate the re-ordered arguments.

Type-1 OWA Operator. Unlike Yager's OWA operator that aggregates crisp values, the type-1 OWA operator is able to aggregate type-1 fuzzy sets with 
uncertain weights, with these uncertain weights being also modelled as type- 1 fuzzy sets. As a generalisation of Yager's OWA operator, and based on Zadeh's Extension Principle [20], a type-1 OWA operator is defined as follows [12]:

Definition 2 Given $n$ linguistic weights $\left\{W^{i}\right\}_{i=1}^{n}$ in the form of type-1 fuzzy sets defined on the domain of discourse $U$, a type-1 OWA operator is a mapping, $\Phi$,

$$
\begin{aligned}
\Phi: \tilde{P}(\mathbb{R}) \times \cdots \tilde{P}(\mathbb{R}) & \longrightarrow \tilde{P}(\mathbb{R}) \\
\left(A^{1}, \cdots, A^{n}\right) & \mapsto Y
\end{aligned}
$$

such that

$$
\begin{aligned}
& \mu_{Y}(y)= \\
& \sum_{\substack{k=1 \\
w_{i} \in U, a_{i} \in X}}^{n} \bar{w}_{i} a_{\sigma(i)}=y
\end{aligned}\left(\begin{array}{l}
\mu_{W^{1}}\left(w_{1}\right) \wedge \cdots \wedge \mu_{W^{n}}\left(w_{n}\right) \\
\wedge \mu_{A^{1}}\left(a_{1}\right) \wedge \cdots \wedge \mu_{A^{n}}\left(a_{n}\right)
\end{array}\right)
$$

where

$$
\bar{w}_{i}=\frac{w_{i}}{\sum_{i=1}^{n} w_{i}}
$$

and

$$
\sigma:\{1, \cdots, n\} \longrightarrow\{1, \cdots, n\}
$$

is a permutation function such that $a_{\sigma(i)} \geq$ $a_{\sigma(i+1)}, \forall i=1, \cdots, n-1$, i.e., $a_{\sigma(i)}$ is the ith highest element in the set $\left\{a_{1}, \cdots, a_{n}\right\}$.

A Direct Approach to performing type-1 OWA operation was suggested in [12]. However, this approach is computationally expensive, which inevitably curtails further applications of the type- 1 OWA operator to real world decision making. So a fast approach to type-1 OWA operations has been developed based on the $\alpha$-level of fuzzy sets [19].

\section{$\alpha$-Level Type-1 OWA Operator.}

Definition 3 Given the $n$ linguistic weights $\left\{W^{i}\right\}_{i=1}^{n}$ in the form of type-1 fuzzy sets defined on the domain of discourse $U$, then for each $\alpha \in U$, an $\alpha$-level type- 1 OWA operator with $\alpha$-level weight sets $\left\{W_{\alpha}^{i}\right\}_{i=1}^{n}$ to aggregate the $\alpha$-level of type- 1 fuzzy sets $\left\{A^{i}\right\}_{i=1}^{n}$ is given as

$$
\begin{aligned}
& \Phi_{\alpha}\left(A_{\alpha}^{1}, \cdots, A_{\alpha}^{n}\right)= \\
& \left\{\frac{\sum_{i=1}^{n} w_{i} a_{\sigma(i)}}{\sum_{i=1}^{n} w_{i}} \mid w_{i} \in W_{\alpha}^{i}, a_{i} \in A_{\alpha}^{i}, \forall i\right\}
\end{aligned}
$$

where $W_{\alpha}^{i}=\left\{w \mid \mu_{W_{i}}(w) \geq \alpha\right\}, A_{\alpha}^{i}=\left\{x \mid \mu_{A_{i}}(x) \geq\right.$ $\alpha\}$, and $\sigma:\{1, \cdots, n\} \rightarrow\{1, \cdots, n\}$ is a permutation function such that $a_{\sigma(i)} \geq a_{\sigma(i+1)}, \forall i=$ $1, \cdots, n-1$, i.e., $a_{\sigma(i)}$ is the ith largest element in the set $\left\{a_{1}, \cdots, a_{n}\right\}$.
According to the Representation Theorem of type-1 fuzzy sets, the $\alpha$-level sets $\Phi_{\alpha}\left(A_{\alpha}^{1}, \cdots, A_{\alpha}^{n}\right)$ obtained via Definition 3 can be used to construct the following type-1 fuzzy set on $\mathbb{R}$

$$
G=\cup_{0<\alpha \leq 1} \alpha \Phi_{\alpha}\left(A_{\alpha}^{1}, \cdots, A_{\alpha}^{n}\right)
$$

with membership function

$$
\mu_{G}(x)=\underset{\alpha: x \in \Phi_{\alpha}\left(A_{\alpha}^{1}, \cdots, A_{\alpha}^{n}\right)_{\alpha}}{\vee} \alpha
$$

Representation Theorem of Type-1 OWA Operators. The two apparently different aggregation results in (11) and (13) obtained according to Zadeh's Extension Principle and the $\alpha$-level of type- 1 fuzzy sets, respectively, are equivalent as proved in [19]:

Theorem 1 Given the $n$ linguistic weights $\left\{W^{i}\right\}_{i=1}^{n}$ in the form of type-1 fuzzy sets defined on the domain of discourse $U$, and the type-1 fuzzy sets $A^{1}, \cdots, A^{n}$, then we have that

$$
Y=G
$$

where $Y$ is the aggregation result defined in (11) and $G$ is the result defined in (13).

Theorem 1 is called the Representation Theorem of Type-1 OWA Operators. Therefore, an effective and practical way of carrying out type-1 OWA operations is to decompose the type-1 OWA aggregation into the $\alpha$-level type-1 OWA operations and then reconstruct it via the above representation theorem. This $\alpha$-level approach has been proved to be much faster than the direct approach [19], so it can be used in real time decision making and data mining applications.

$\alpha$-Level Type-1 OWA of Fuzzy Numbers. When the linguistic weights and the aggregated sets are fuzzy number, the $\alpha$-level type-1 OWA operator produces closed intervals [19]:

Theorem 2 Let $\left\{W^{i}\right\}_{i=1}^{n}$ be fuzzy numbers on $U$ and $\left\{A^{i}\right\}_{i=1}^{n}$ be fuzzy numbers on $\mathbb{R}$. Then for each $\alpha \in U, \Phi_{\alpha}\left(A_{\alpha}^{1}, \cdots, A_{\alpha}^{n}\right)$ is a closed interval.

Based on this result, the computation of the type1 OWA output according to (13), G, reduces to compute the left end-points and right end-points of the intervals $\Phi_{\alpha}\left(A_{\alpha}^{1}, \cdots, A_{\alpha}^{n}\right)$ :

$$
\Phi_{\alpha}\left(A_{\alpha}^{1}, \cdots, A_{\alpha}^{n}\right)_{-} \text {and } \Phi_{\alpha}\left(A_{\alpha}^{1}, \cdots, A_{\alpha}^{n}\right)_{+},
$$

where $A_{\alpha}^{i}=\left[A_{\alpha-}^{i}, A_{\alpha+}^{i}\right], W_{\alpha}^{i}=\left[W_{\alpha-}^{i}, W_{\alpha+}^{i}\right]$.

For the left end-points, we have

$$
\begin{aligned}
& \Phi_{\alpha}\left(A_{\alpha}^{1}, \cdots, A_{\alpha}^{n}\right)_{-}= \\
& \min _{W_{\alpha-}^{i} \leq w_{i} \leq W_{\alpha+}^{i}} \sum_{i=1}^{n} w_{i} a_{\sigma(i)} / \sum_{i=1}^{n} w_{i} \\
& A^{i} \leq a_{i} \leq A^{i}
\end{aligned}
$$


while for the right end-points, we have

$$
\begin{aligned}
& \Phi_{\alpha}\left(A_{\alpha}^{1}, \cdots, A_{\alpha}^{n}\right)_{+}= \\
& \quad \max \\
& W_{\alpha-}^{i} \leq w_{i} \leq W_{\alpha+}^{i} \sum_{i=1}^{n} w_{i} a_{\sigma(i)} / \sum_{i=1}^{n} w_{i} \\
& A_{\alpha-}^{i} \leq a_{i} \leq A_{\alpha+}^{i}
\end{aligned}
$$

It can be seen that (15) and (16) are programming problems. Solutions to these problems, so that the type-1 OWA aggregation operation can be performed efficiently, are available from [19].

\section{Centroid of Type-2 Fuzzy Sets}

The definition of the centroid of a type- 1 fuzzy set $A$ in $X$, also referred to as the centre of gravity or centre of mass, requires the universe of discourse to be a subset of the set of real numbers. Therefore, from now on we will assume that the domain of the type-1 fuzzy set is of such type.

The centroid for a continuum universe of discourse $X$ is defined as

$$
C_{A}=\frac{\int_{x} x \cdot \mu_{A}(x) d x}{\int_{x} \mu_{A}\left(x_{i}\right)}
$$

The centroid when the domain $X$ is discretised into $n$ points is

$$
C_{A}=\frac{\sum_{i=1}^{n} x_{i} \cdot \mu_{A}\left(x_{i}\right)}{\sum_{i=1}^{n} \mu_{A}\left(x_{i}\right)}
$$

Note that in this discrete form of the centroid of a type- 1 fuzzy set it is true that $x_{1}<x_{2}<\ldots<x_{n}$.

Centroid of Type-2 Fuzzy Sets. As we mentioned above, the defuzzification stage for type-2 fuzzy sets consists of two parts: (i) type-reduction, and (ii) defuzzification proper [3]. The challenging and complex step when deriving the centroid of a type-2 fuzzy sets is obviously the computation of the TRS. The TRS is defined via the application of Zadeh's Extension Principle, and only after the universe of discourse is being discretised.

Definition 4 The TRS associated to a type-2 fuzzy sets $\tilde{A}$ with domain $X$ discretised into $n$ points is

$$
\begin{aligned}
C_{\tilde{A}}=\int_{u_{1} \in J_{x_{1}}} \ldots \int_{u_{n} \in J_{x_{n}}}\left[\mu_{\tilde{A}}\left(x_{1}\right)\left(u_{1}\right) * \ldots *\right. \\
\left.\mu_{\tilde{A}}\left(x_{n}\right)\left(u_{n}\right)\right] / \frac{\sum_{i=1}^{n} x_{i} \cdot u_{i}}{\sum_{i=1}^{n} u_{i}}
\end{aligned}
$$

Note that the TRS is a type- 1 fuzzy set in $U$. Again in this case, we have $x_{1}<x_{2}<\ldots<x_{n}$.
The type reduction stage requires the application of a t-norm $(*)$ to the secondary membership degrees. Because the product t-norm does not produce meaningful results for type-2 fuzzy sets with general secondary membership functions, the the minimum t-norm $(\wedge)$ is used [3, pp. 251-253].

Centroid of an Interval Type-2 Fuzzy Sets. In the case of $\tilde{A}$ being an interval type- 2 fuzzy set, i.e. $\mu_{\tilde{A}}(x)(u)=1 \forall x, u$, we have that the TRS is the crisp set

$$
C_{\tilde{A}}=\int_{u_{1} \in J_{x_{1}}} \cdots \int_{u_{n} \in J_{x_{n}}} 1 / \frac{\sum_{i=1}^{n} x_{i} \cdot u_{i}}{\sum_{i=1}^{n} u_{i}}
$$

\section{Relationship between the TRS of a type-2 fuzzy set and the type-1 OWA operator}

We note that the type-1 fuzzy set derived after the application of a type-1 OWA operator can be rewritten as follows:

$$
\begin{array}{r}
Y=\int_{w_{1} \in S\left(W^{1}\right)} \ldots \int_{w_{n} \in S\left(W^{n}\right)} \int_{a_{1} \in S\left(A^{1}\right)} \ldots \int_{a_{n} \in S\left(A^{n}\right)} \\
{\left[\mu_{W^{1}}\left(w_{1}\right) \wedge \cdots \wedge \mu_{W^{n}}\left(w_{n}\right) \wedge \mu_{A^{1}}\left(a_{1}\right) \wedge \cdots \wedge\right.} \\
\left.\wedge \mu_{A^{n}}\left(a_{n}\right)\right] / \frac{\sum_{i=1}^{n} w_{i} a_{\sigma(i)}}{\sum_{i=1}^{n} w_{i}}
\end{array}
$$

where $S\left(W^{i}\right)$ and $S\left(A^{1}\right)$ are the support sets of $W^{i}$ and $A^{i}$, respectively, for all $i=1, \ldots, n$, i.e.

$$
S\left(W^{i}\right)=\left\{w \in U \mid \mu_{W^{i}}(w)>0\right\} ;
$$

and

$$
S\left(A^{i}\right)=\left\{a \in X \mid \mu_{A^{i}}(a)>0\right\} .
$$

Given a type-2 fuzzy set $\tilde{A}$, for a group of discretised points on the domain $X: x_{1}<x_{2}<\ldots<x_{n}$, suppose the type- 1 fuzzy sets $A^{1}, \ldots, A^{n}$ in expression (21) be chosen as $A^{1}=x_{1}, \ldots, A^{n}=x_{n}$, i.e, the aggregated objects are specially set to be singleton type-1 fuzzy sets, while the uncertain weights $W^{1}, \ldots, W^{n}$ are set to be $W^{1}=\mu_{\tilde{A}}\left(x_{n}\right), \ldots, W^{n}=$ $\mu_{\tilde{A}}\left(x_{1}\right)$. Then expression $(21)$ reduces to

$$
\begin{array}{r}
Y=\int_{w_{1} \in J_{x_{n}}} \ldots \int_{w_{n} \in J_{x_{1}}}\left[\mu_{W^{1}}\left(w_{1}\right) * \ldots *\right. \\
\left.\mu_{W^{n}}\left(w_{n}\right)\right] / \frac{\sum_{k=1}^{n} w_{i} x_{\sigma(i)}}{\sum_{i=1}^{n} w_{i}}
\end{array}
$$

that is to say,

$$
\begin{array}{r}
Y=\int_{u_{1} \in J_{x_{1}}} \ldots \int_{u_{n} \in J_{x_{n}}}\left[\mu_{\tilde{A}}\left(x_{1}\right)\left(u_{1}\right) * \ldots *\right. \\
\left.\mu_{\tilde{A}}\left(x_{n}\right)\left(u_{n}\right)\right] / \frac{\sum_{i=1}^{n} u_{i} \cdot x_{i}}{\sum_{i=1}^{n} u_{i}}
\end{array}
$$


This expression coincides with the TRS associated to a type- 2 fuzzy sets $\tilde{A}$ with domain $X$ discretised into $n$ points as per the expression (19) given in Definition 4.

In the case of an interval type- 2 fuzzy set $\tilde{A}$ with domain $X$ discretised into $n$ points, its TRS can be expressed as

$$
C_{\tilde{A}}=\left\{\frac{\sum_{i=1}^{n} x_{i} \cdot u_{i}}{\sum_{i=1}^{n} u_{i}} \mid u_{i} \in J_{x_{i}}\right\}
$$

In this case, the primary membership of $x_{i}, J_{x_{i}}$, is a closed interval and therefore $C_{\tilde{A}}$ is also closed. What we are showing here is that this formulation of the TRS of an interval type- 2 fuzzy set $\tilde{A}$ with domain $X$ discretised into $n$ points is equivalent to the one we obtained for the $\alpha$-level type-1 OWA operator as per Definition 3. Indeed, when the input of an $\alpha$-level type-1 OWA operator, $\left(A_{\alpha}^{1}, \cdots, A_{\alpha}^{n}\right)$, reduces to a singleton point in the space $\mathbb{R}^{n}$, expression (12) reduces to (24), and therefore both mathematical representations are equivalent. So the centroid of an interval type-2 fuzzy set is a special case of $\alpha$-level type-1 OWA operator.

\section{Conclusions}

We have shown that the apparent disparate problems consisting in the computation of the TRS of a type- 2 fuzzy set and the type-1 OWA aggregation of type-1 fuzzy sets are closely related. In essence, both problems are aggregation problems, and both are defined via the application of Zadeh's Extension Principle. However, we have shown that the Type1 OWA Representation Theorem, by which type-1 OWA operators can be decomposed into a series of $\alpha$-level type-1 OWA operators, is the key result that allows us to prove that the TRS of a type- 2 fuzzy sets is a particular case of a type-1 OWA operator. In particular, we have also proved that the centroid of an interval type-2 fuzzy sets is a particular case of an $\alpha$-level type-1 OWA operator.

The main consequence of the above result is that a fast and efficient method to compute the centroid of a general type- 2 fuzzy set could become possible, what in future could imply an increase use of general type-2 fuzzy sets for real applications.

\section{References}

[1] R. R. Yager, On Ordered Weighted Averaging Aggregation Operators in Multi-criteria Decision Making, IEEE Trans. on Systems, Man and Cybernetics, 18:83-190, 1988.

[2] L. A. Zadeh, Outline of a New Approach to the Analysis of Complex Systems and Decision Processes, IEEE Trans. on Systems, Man and Cybernetics, 3:28-44, 1973.

[3] J. M. Mendel, Uncertain Rule-Based Fuzzy Logic Systems: Introduction and New Directions. Prentice-Hall PTR, 2001.
[4] J. M. Mendel and H. Wu, New Results about the Centroid of an Interval Type-2 Fuzzy Set, Including the Centroid of a Fuzzy Granule, Information Sciences, 17:360-377, 2007.

[5] J. T. Starczewski, On Defuzzification of Interval Type-2 Fuzzy Sets. In proceedings of the $9^{\text {th }}$ International Conference on Artificial Intelligence and Soft Computing (ICAISC 2008), Lecture Notes in Computer Science 5097, pages 333-340, Springer-Verlag, 2008.

[6] S. Greenfield, F. Chiclana, S. Coupland and R. I. John, The Collapsing Method of Defuzzification for Discretised Interval Type-2 Fuzzy Sets, Information Sciences, 179(13):2055-2069, 2009.

[7] D. R. Wu and J. M. Mendel, Enhanced KarnikMendel Algorithms, IEEE Transactions on Fuzzy Systems, 17:923-934, 2009.

[8] E. A. Jammeh, M. Fleury, C. Wagner, H. Hagras and M. Ghanbari, Interval Type-2 fuzzy Logic Congestion Control for Video Streaming Across IP Networks, IEEE Transactions on Fuzzy Systems, 17:1123-1142, 2009.

[9] E. Kayacan, O. Kaynak, R. Abiyev, J. Tǒrresen, M. Hǒvin and K. Glette, Design of an Adaptive Interval Type-2 Fuzzy Logic Controller for the Position Control of a Servo System with an Intelligent Sensor, In Proceedings of FUZZ-IEEE 2010, pages 1125-1132, Barcelona, Spain, 2010.

[10] L. Leottau and M. Melgarejo, Implementing an Interval Type-2 Fuzzy Processor onto a DSC 56F8013, In Proceedings of FUZZ-IEEE 2010, pages 1939-1942, Barcelona, Spain, 2010.

[11] C. Leal-Ramirez, O. Castillo, P. Melin and A. Rodriguez-Diaz, Simulation of the Bird Agestructured Population Growth Based on an Interval Type-2 Fuzzy Cellular Structure, Information Sciences, 181(3):519-535, 2011

[12] S -M. Zhou, F. Chiclana, R. I. John and J. M. Garibaldi, Type-1 OWA Operators for Aggregating Uncertain Information with Uncertain Weights Induced by Type-2 linguistic Quantifiers, Fuzzy Sets and Systems, 159 (24):3281$3296,2008$.

[13] F. Herrera, E. Herrera-Viedma and F. Chiclana, A Study of the Origin and Uses of the Ordered Weighted Geometric Operator in Multicriteria Decision Making, International Journal of Intelligent Systems,18(6):689-707, 2003.

[14] F. Chiclana, E. Herrera-Viedma, F. Herrera and S. Alonso, Induced Ordered Weighted Geometric Operators and Their Use in the Aggregation of Multiplicative Preference Relations, International Journal of Intelligent Systems, 19(3):233-255, 2004.

[15] F. Chiclana, E. Herrera-Viedma, F. Herrera and S. Alonso, Some Induced Ordered Weighted Averaging Operators and Their Use for Solving Group Decision-making Problems Based on Fuzzy Preference Relations, European Journal of Operational Research, 182(1):383-399,2007. 
[16] S. -M. Zhou and J. Q. Gan, Constructing Accurate and Parsimonious Fuzzy Models with Distinguishable Fuzzy Sets Based on an Entropy Measure, Fuzzy Sets and Systems, 157(8): 10571074, 2006.

[17] S. -M. Zhou and J Q. Gan, Constructing L2-SVM-based Fuzzy Classifiers in Highdimensional Space with Automatic Model Selection and Fuzzy Rule Ranking, IEEE Trans. on Fuzzy Systems 15 (3): 398-409, 2007.

[18] S. -M. Zhou and J. Q. Gan, Extracting TakagiSugeno Fuzzy Rules with Interpretable Submodels Via Regularization of Linguistic Modifiers, IEEE Transactions on Knowledge and Data Engineering, 21(8):1191-1204, 2009.

[19] S. -M. Zhou, F. Chiclana, R. I. John and J. M. Garibaldi, Alpha-Level Aggregation: A Practical Approach to Type-1 OWA Operation for Aggregating Uncertain Information with Applications to Breast Cancer Treatments, IEEE Transactions on Knowledge and Data Engineering doi: 10.1109/TKDE.2010.191

[20] L. A. Zadeh, The Concept of a Linguistic Variable and its Application to Approximate Reasoning - I, Information Sciences, 8:199-249, 1975 . 